THERAPEUTIC TARGETS

\section{Withdrawal symptoms}

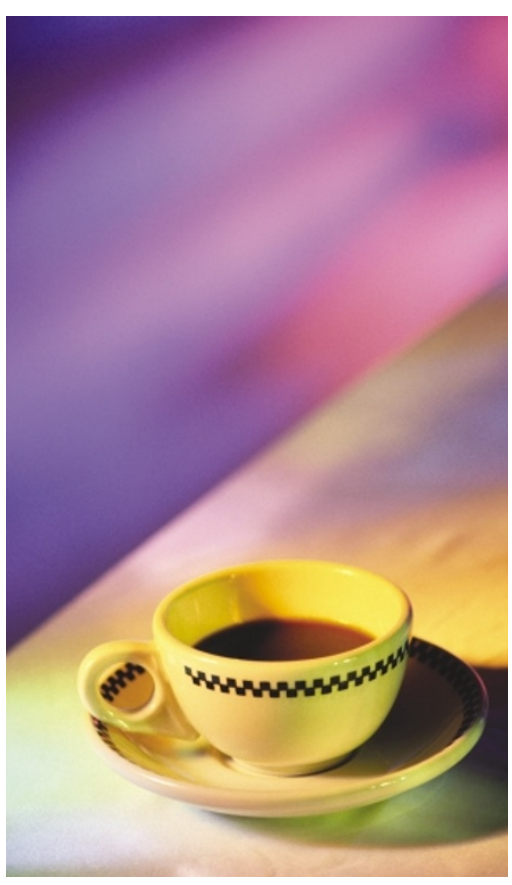

We all know how tetchy we can be after skipping that vital cup of morning coffee; well, cancer cells can become addicted to stimulants, too. Two papers in the August 28 issue of Proceedings of the National Academy of Sciences reveal that cancer cells with a hyperactive phosphatidylinositol 3-kinase (PI3K) pathway become reliant on it to activate FRAP1 (mTOR), a kinase that regulates ribosome biogenesis. This makes these cells more sensitive to rapamycin - a drug that is already on the market as an immunosuppressant.

The PI3K pathway is frequently activated in cancer cells: amplification of PI3K itself causes the overproduction of 3-phosphorylated inositol lipids, which activate a protein kinase called AKT. The same effect can be achieved by loss of PTEN, the phosphatase that removes the 3-phosphate from inositol lipids, thereby inactivating them as second messengers. An obvious way to block this pathway would be to inhibit AKT, but no AKT-selective inhibitors have yet been developed.

FRAP1 is phosphorylated and activated by AKT, although it can also be activated independently of AKT. Might blocking FRAP1 inhibit the growth of cells that are overdoing the 3-phosphorylated inositol lipids? Charles Sawyers and colleagues investigated this possibility in $\mathrm{Pten}^{+/+}$and $\mathrm{Pten}^{-1}$ mouse embryonic fibroblasts, and in a panel of human cancer cell lines with known PTEN status, using CCI-779 — an esterified form of rapamycin. Nanomolar concentrations of CCI-779 blocked proliferation (determined by ${ }^{3} \mathrm{H}$-thymidine uptake) in all the $P T E N^{-/}$cells. But is this due to FRAP1 inhibition? The activity of S6 kinase, a downstream target of FRAP1, was upregulated in $P T E N^{-1-}$ cells by both increased protein production and inceased phosphorylation, but treatment with CCI-779 reduced S6 kinase to undetectable levels.
What effect does CCI-779 have in vivo? Tumour growth was inhibited in both $P T E N^{+/+}$and $P T E N^{/-}$tumours that were grown in immunodeficient mice, but the $P T E N^{-1-}$ tumours were much more sensitive to the drug. This was due to FRAP1 inhibition because phosphorylation of S6 kinase, which was increased in $\mathrm{PTEN}^{-/}$tumour explants from untreated animals compared with $P T E N^{+/+}$explants, was almost undetectable in CCI-779treated animals. CCI-779 reduced tumour volume by decreasing cell division and reducing cell size, but it didn't stimulate apoptosis.

Analysis of two downstream targets of FRAP1, S6 kinase and 4E-BP1, showed that CCI-779 blocked FRAP1 signalling regardless of PTEN status, so one explantion for why $P T E N^{-1-}$ cells are more sensitive to the drug than $\mathrm{PTEN}^{+/+}$cells is that they've become 'hooked' on PI3K signalling to activate cell division, even though there are other pathways that can do the job.

In the second paper, Ramon Parsons and colleagues describe the effects of CCI-779 in Pten ${ }^{+/-}$mice,

\title{
Keep young and beautiful
}

\author{
The quest for immortality has long been \\ pursued by humans - as yet to no avail - \\ but it is also a crucial step on the path to \\ tumour formation, and cancer cells will use \\ any means at their disposal to achieve this \\ end. Anil Rustgi and colleagues, reporting \\ in the September issue of The Journal of \\ Clinical Investigation, provide a model for \\ how this can be achieved by the alternative \\ (ALT) pathway - a telomerase- \\ independent mechanism. \\ The authors analysed the effects on \\ cellular immortality of genetic changes \\ that occur in oral-oesophageal squamous \\ cell carcinoma, using oral keratinocytes as \\ a model. They first overexpressed the cyclin \\ D1 oncogene, the most common alteration \\ in this cancer type. The effect was twofold: \\ the cell-cycle distribution changed to \\ include $20 \%$ more cells in S phase, and cells \\ had an extended lifespan, undergoing 80 \\ population doublings before entering \\ senescence, rather than 40 . \\ Combining this with a dominant-negative
}

TP53 mutation - another frequent genetic change in cancer - exacerbated the cellgrowth phenotype. These cells now had a lifespan of greater than 160 population doublings, and showed no signs of arrest. So, what was the mechanism of immortality?

Two mechanisms can facilitate immortality: activation of telomerase and activation of the ALT pathway. Rustgi and colleagues did not find evidence of the first possibility, as telomerase activity was absent from both the parent oral keratinoctyes and the ones expressing cyclin D1 and dominant-negative p53. Consistent with a lack of telomerase activity, telomere length - measured by Southern blotting with a telomere-specific probe - was initially shown to decrease with cell age. However, keratinocytes expressing cyclin D1 and dominantnegative p53 were found to have longer, albeit heterogeneous, telomeres by 160 population doublings. The heterogeneity, coupled with a karyotypic analysis that revealed aneuploidy and chromosomal changes, supported the hypothesis that the ALT pathway, which maintains telomeres through non-homologous recombination, was involved.

Interestingly, injection of these cells into nude mice did not result in tumour formation, indicating that they were not malignantly transformed. These keratinocytes therefore provide an interesting model that will allow the investigation of two problems: what additional changes are needed for transformation, and what is the mechanism by which the ALT pathway stabilizes telomeres? Emma Greenwood

6) References and links ORIGINAL ReSEARCh PAPER Opitz, O. G. et al. Cyclin D1 overexpression and p53 inactivation immortalize priman oralkeratinocytes by a tomerase independent mechanism. J. Clin Invest. 108, 725-732 (2001) J. Clin. Invest. 108, 725-732 (2001)

FURTHER READING Reddel, R. R. An alternative lifestyle for immortalized oral keratinocytes. J. Clin. Invest. 108, 665-667 (2001)

WEB SITE

Anil Rustgi's lab:www.uphs.upenn.edu/abramson/ rustgi.html 
which develop endometrial cancers and phaeochromocytomas that frequently show loss of heterozygosity of the second Pten allele. Treatment of $\mathrm{Pten}^{+/-}$mice with CCI-779 for 20 weeks significantly reduced tumour volume and, again, this seemed to be due to growth inhibition rather than cell killing.

So, by preventing $\mathrm{PTEN}^{-/-}$cancers - which include prostate and endometrial tumours and glioblastomas - from activating FRAP1 through the PI3K pathway, we might be able to halt their growth. AKT remains an enticing therapeutic target as its inhibition should block PI3Kmediated growth and survival signals. But, in the meantime, CCI-779 is worthy of clinical investigation.

(2) References and links Cath Brooksbank ORIGINAL RESEARCH PAPERS Neshat, M. S. et al. Enhanced sensitivity of PTEN-deficient tumors to inhibition of FRAP/mTOR. Proc. Natl Acad. Sci. USA 98, 10314-10319 (2001) | Podsypanina, K. et al. An inhibitor of mTOR reduces neoplasia and normalizes p70/S6 kinase activity in Pten ${ }^{+/-}$mice. Proc. Natl Acad. Sci. USA 98, 10320-10325 (2001) Wroc. Nat/Acad SITE

WEB SITE
Biocarta: regulation of elF4e and p70 $\$ 6$ kinase http://www.biocarta.com/pathfiles/eif4Pathway.asp

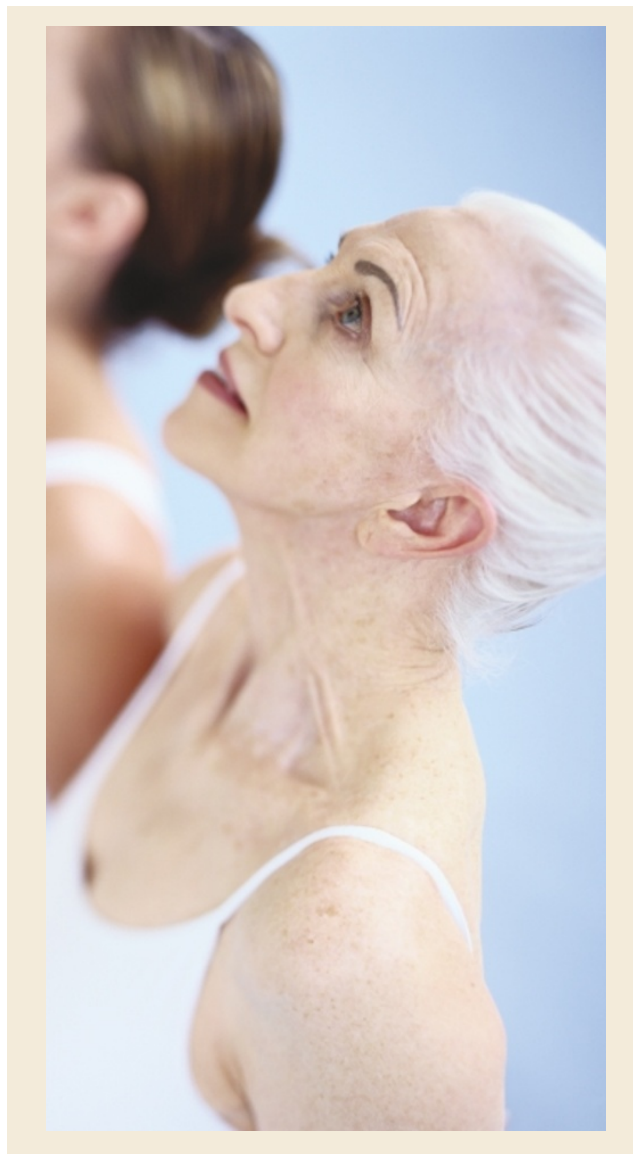

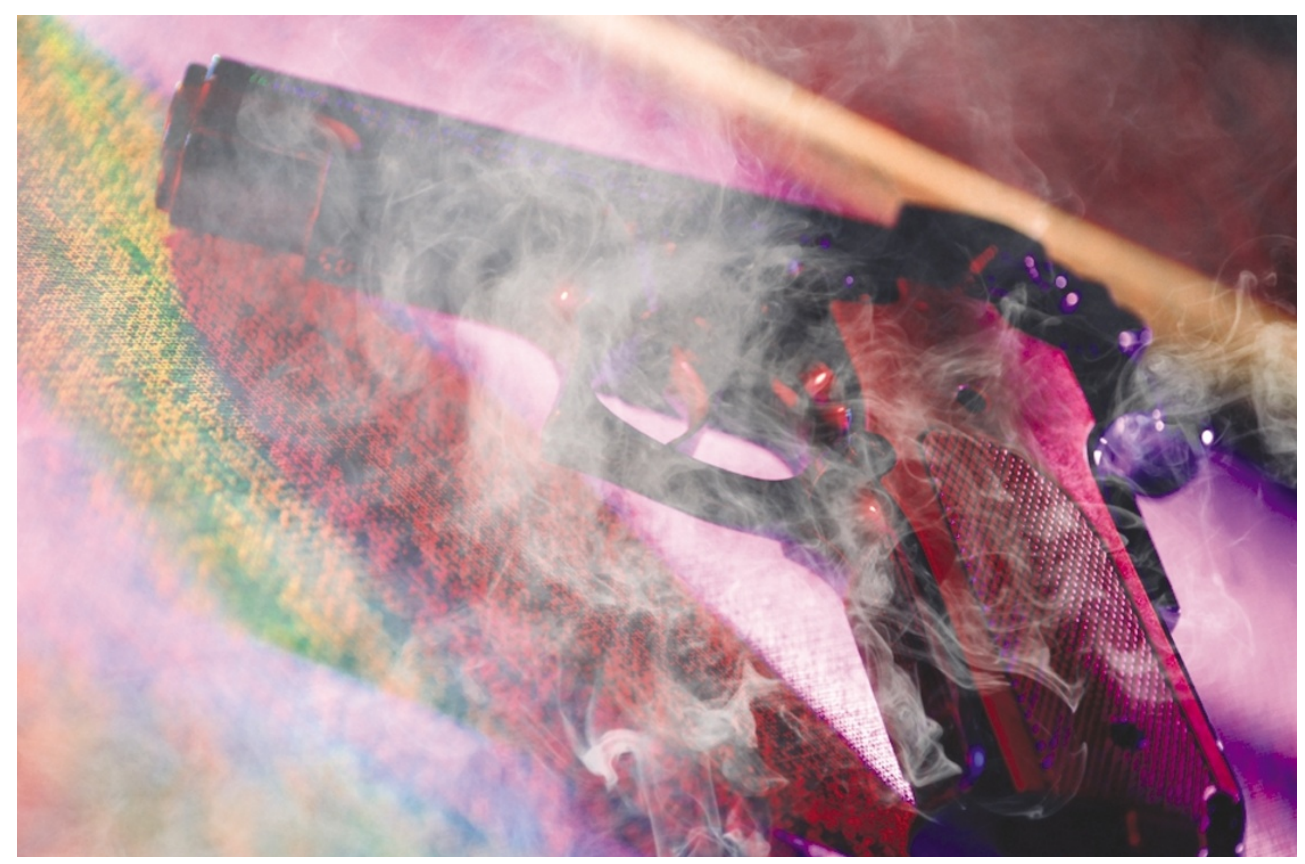

\section{CHEMOTHERAPY}

\section{Smoking gun}

5-fluorouracil (5-FU) has proved to be one of the most effective chemotherapeutics for colon cancer. It induces apoptosis in rapidly dividing cells, but little is known about the molecular mechanism by which it causes them to self-destruct. In the October issue of Nature Medicine, Bert Vogelstein and colleagues report that 5-FU depends on ferredoxin reductase (FR) — an 'electron gun' - to kill cells through oxidative stress.

Previous studies have shown that apoptosis induction by 5 -FU involves the transcription factor p53, but what else? Vogelstein's group used serial analysis of gene expression (SAGE) to identify 5-FUresponsive genes. By comparing gene-expression patterns between 5 -FU-sensitive TP5 $3^{+/+}$human colon cancer cells and 5-FU-resistant $T P 53^{-/-}$cells, they could isolate genes that were only transcribed in association with $\mathrm{p} 53$-mediated cell death.

SAGE libraries were constructed from mRNA purified from each cell type after 18 hours of treatment with 5-FU - long enough for transciption to be activated, but well before the cellular apoptotic programme starts. Surprisingly, only a few genes were induced by 5 -FU in a p53-dependent manner. One unexpected finding was that the gene that encodes FR, which had not been previously associated with p53 or apoptosis, was upregulated within 3 hours of drug treatment, indicating that it could be a direct transcriptional target of p53. Sure enough, the authors found that the FR gene promoter contained a p53-binding site and that its expression was indeed activated after 5-FU treatment. Furthermore, p53 activates FR expression in three different colon cancer cell lines.

Next, Vogelstein's group investigated the biological functions of FR. The colon cancer cell line studied carried three copies of the gene that encodes FR, but deleting all three copies was lethal, indicating its essential role in cell survival.
Disruption of two of the three copies reduced 5-FU-induced apoptosis, so it is likely to be an important mediator of cell death.

But how does FR contribute to the apoptotic pathway? FR sits on the matrix side of the inner mitochondrial membrane, where it is involved in transferring electrons from reduced nicotinamide adenine dinucleotide phosphate (NADPH) to substrates such as cholesterol. Under substrate-limiting conditions, FR releases electrons, which can then generate reactive oxygen species (ROS) such as superoxide. So, too much FR and not enough substrate can generate oxidative stress. Several previous studies had already implicated mitochondrialderived ROS in p53-mediated cell death, so FR might be one of the missing links in this process.

To prove that FR's role in p53-mediated cell death involves the generation of oxidative stress, the authors used a chemical indicator to measure ROS production after 5-FU treatment. Colon cancer cells with intact TP53 and FR genes produced high levels of ROS after exposure to 5-FU, whereas colon cancer cells with disruptions in TP53 or FR did not. Furthermore, a pharmacological antioxidant prevented 5-FU-induced apoptosis, showing that killing cells by $5-\mathrm{FU}$ depends on ROS generation.

So, is this definitive proof that 5-FU induces toxicity strictly through p 53 and generation of oxidative stress? Apparently not, as the authors showed that neither disruption of TP53 nor FR allowed colon cancer cells to survive 5-FU treatment when measured by clonogenic assays, a more stringent test of chemosensitivity than the apoptosis assays used in this study. Therefore, 5-FU seems to have other toxic effects in cells, not involving p53, that await discovery.

Kristine Novak

\section{(2) References and links}

ORIGINAL RESEARCH PAPER Hwang, P. M. etal. Ferredoxin reductase affects p53-dependent, 5-fluorouracil-induced apoptosis in colorectal cancer cells. Nature Med. 7, 1111-1117 (2001)

FURTHER READING Vogelstein, B., Lane, D. \& Levine, A. J. Surfing the p53 network. Nature 408, 307-310 (2000)

Bert Vogelstein's lab:

http://www.med.jhu.edu/pharmacology/pages/faculty/vogelstein.html

The SAGE home page: www.sagenet.org/ 